\title{
THE FEASIBILITY OF EMPLOYING IEEE802.11P IN ELECTRONIC-BASED CONGESTION PRICING ZONE: A COMPARATIVE STUDY WITH RFID
}

\author{
Alireza Marefat ${ }^{1}$, Rafidah Md Noor ${ }^{2}$, Nor Badrul Anuar ${ }^{3}$, Nornazlita Hussin $^{4}$ \\ 1,2,3,4 Department of Computer Science and Information Technology \\ University of Malaya, Kuala Lumpur, Malaysia
}

Email: ${ }^{1}$ alimarefat69@siswa.um.edu.my, ${ }^{2}$ fidah@um.edu.my, ${ }^{3}$ badrul@um.edu.my, ${ }^{4}$ nazlita@um.edu.my

\begin{abstract}
Congestion pricing has been defined as the best solution to efficiently alleviate the traffic congestion in a designated area. Generally, the basis of congestion pricing zone (CPZ) systems comprised of the following three main phases: vehicle detection, data communication, and enforcement. In terms of data communication, the majority of the existing systems utilize Radio Frequency IDentification (RFID) which contains several limitations such as a low transmission range, low data rate, communication drawbacks like delay, tag and reader collisions, signal fading, and interception. In this particular context, it would be very constructive to take advantage of a Vehicular ad hoc Network (VANET) that is based on the Dedicated Short Range Communication (DSRC) method to design a pervasive-congestion pricing system. This study aims to design an optimized CPZmanagement model using VANET to solve the existing mainstream problems. The proposed approach is evaluated and validated using a simulation environment and a selection of tools including NS-2, SUMO, and TraNS. The experimental results from two different scenarios (i.e. using RFID-based RSU and DSRC-based $R S U)$ were attained after considering the number, velocity, and occupancy of the contributed vehicles, and the traffic flow. The obtained results show that DSRC outperformed RFID in terms of occupancy, traffic flow, and velocity by an average of $8.27 \%, 22 \%$, and $13.75 \%$, respectively. Consequently, the DSRC employment under VANET infrastructure not only enhances data-communication, but also reduces traffic congestion around CPZ.
\end{abstract}

Keywords: Congestion pricing, Vehicular ad Hoc Network (VANET), Radio Frequency IDentification (RFID), Dedicated Short Range Communication (DSRC), Intelligent Transportation System (ITS)

\subsection{INTRODUCTION}

After many decades of automobile usage, metropolises throughout the world face the critical problem of traffic congestion that is especially problematic in central-city areas; the underlying cause is defined by the continuing increase of both populations and the transportation needs of people in daily life. Accordingly, the problems of crowded streets and traffic congestion have become a critical concern of city councils. Congestion issues not only has an impact on government, but also directly affect drivers and passengers in many cases through longer trip times, vehicular queuing, slow speeds, and the air pollution from fuel emissions, among others [1]. Solutions for congestion elimination have therefore been investigated by economists, authorities, and academics; accordingly, researchers found that congestion pricing (CP) is one of the best solutions to efficiently reduce the amount of vehicles in a designated area [2]. For many years, CP remained as just an idea, but the interest in the concept gradually spread beyond academia and it was eventually practiced to a limited extent. Since the late 1980s, CP-implementation attempts have occurred; currently, the foremost functioning CP systems are the London Congestion Charge [3], the Stockholm cordon charge [4], Singapore's Electronic Road Pricing (ERP) system [5], and the Norwegian toll ring [6], all of which are compared in the Literature section. All of the previously mentioned CP systems provide the same advantages and achievements such as the redirection of people into public-transportation systems, the provision of a source of funds to improve public transport, the enhancement of road facilities, the decrease of emissions in city atmospheres, the alleviation of traffic congestion, and the increases of traffic flow and general speed in most congested areas [7].

Basically, the functioning of a CPZ system is based on the following three main phases: 1. Vehicle detection for the detection of vehicles, their coordinates, and/or their mileage, as well as other useful information such as the number of axles, type of vehicle, and time of day. 2. Data communication to allow communication between 
road-side units (RSU) and an in-vehicle unit (IVU). 3. Enforcement to conduct functions like charging and violation enforcement in the case of any type of violation [8]. These phases are illustrated in Fig. 1.

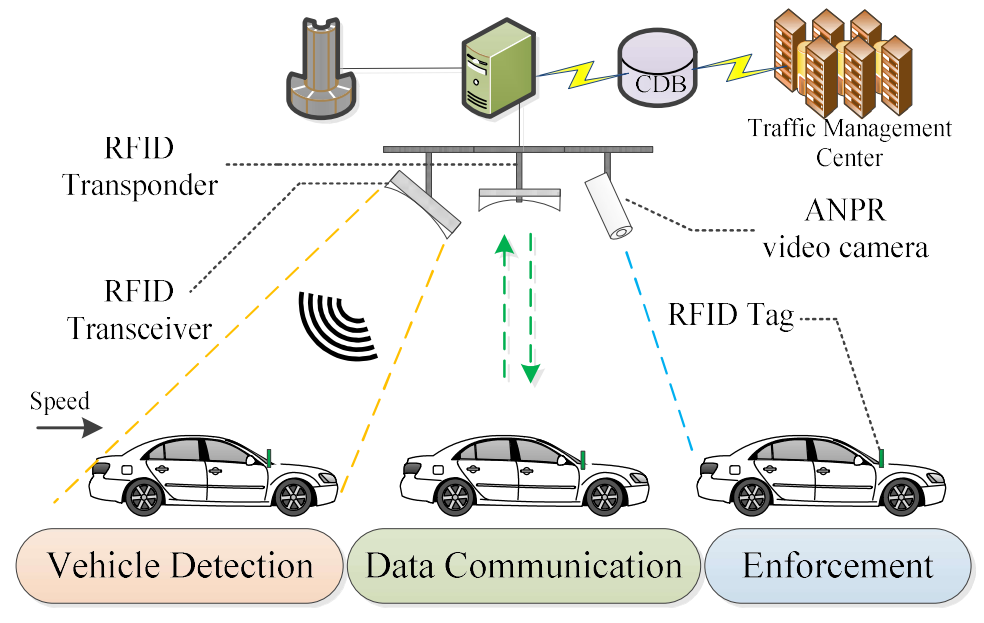

Fig. 1: Three main operational phases of $\mathrm{CPZ}$

There are several shortcomings for each of the existing system to be discussed in the next section. This article discusses the design weaknesses and technological problems of CPZ systems with a focus on communication technologies. The majority of existing systems use Radio Frequency Identification (RFID) technology to accomplish the second phase of a CPZ system (i.e. data communication). RFID is a technology for automated identification and object tracking, whereby people attach tags to objects for the purpose of tracking. An RFID tag is a small microchip that is designed for wireless data transmission in response to an inquiry by an RFID reader. RFID devices can be classified into the following two classes: active and passive [9]. Active tags need a power source (e.g. power supplier or embedded battery) to operate, while passive tags have a limited lifetime and number of read operations based on their stored energy. Nonetheless, various applications choose a passive RFID system in an ultra-high frequency (UHF; $860 \mathrm{MHz}$ to $2.45 \mathrm{GHz}$ ) because of a low tag price and a relatively long radio range (up to $10 \mathrm{~m}$ ). In the passive RFID system, the RFID reader emits RF radio waves that provoke a signal back from the tag; the RFID tag that stored its ID in the memory works without an external power source. More precisely, upon receiving the radio waves, the tag absorbs energy and pumps back the waves that are modulated with its own ID signature. The most notable advantage of passive RFID is its battery-free operation [10].

The utilization of the Vehicular ad hoc Network (VANET), which is a subdirectory of the Intelligent Transportation System (ITS) [8] concept that employs Dedicated Short Range Communication (DSRC) as a communication method, can address the majority of the previously stated problems. VANET is a self-organized network composed of mobile nodes that are connected with wireless links where the vehicles act as nodes [11] [12]. In VANETs, each vehicle plays the roles of sender, receiver, and router to broadcast data to the vehicular network that then uses the data to ensure a safe, free-flow of traffic [13]. For communication to take place between RSUs and vehicles, the vehicles must be equipped with an on-board unit (OBU) that enables the formation of short-range wireless ad hoc networks. In addition, vehicles must be equipped with hardware that permits detailed position information such as the Global Positioning System (GPS) or a Differential Global Positioning System (DGPS) receiver. On the other side, fixed RSUs that are linked to the backbone network must be mounted to facilitate communication [14]. As Fig. 2 illustrates, the communication configurations in VANET contains Vehicle-to-Vehicle (V2V), Vehicle-to-Infrastructure (V2I), and inter-roadside communications that rely on accurate information regarding the surrounding environment; this information is dependent upon the employment of accurate positioning systems and effective communication protocols for the transferal of information.

In 2003, the Federal Communication Commission (FCC) established DSRC service, a communication service for private and public safety that operates at a frequency range between $5.850 \mathrm{GHz}$ and $5.925 \mathrm{GHz}$ [15]. IEEE developed a Wireless Access in Vehicular Environments (WAVE) standard, or IEEE802.11p [16], to provide the 
DSRC for VANET communications. A multi-channel spectrum system has been developed for DSRC that encompasses seven channels and provides $10 \mathrm{MHz}$ of bandwidth per channel, six of which are Service Channels (SCHs) and one is identified as the Control Channel (CCH). SCHs are utilized for non-safety and WAVE-mode messages or services, whereas $\mathrm{CCH}$ is used for safety messages $[17,18]$.

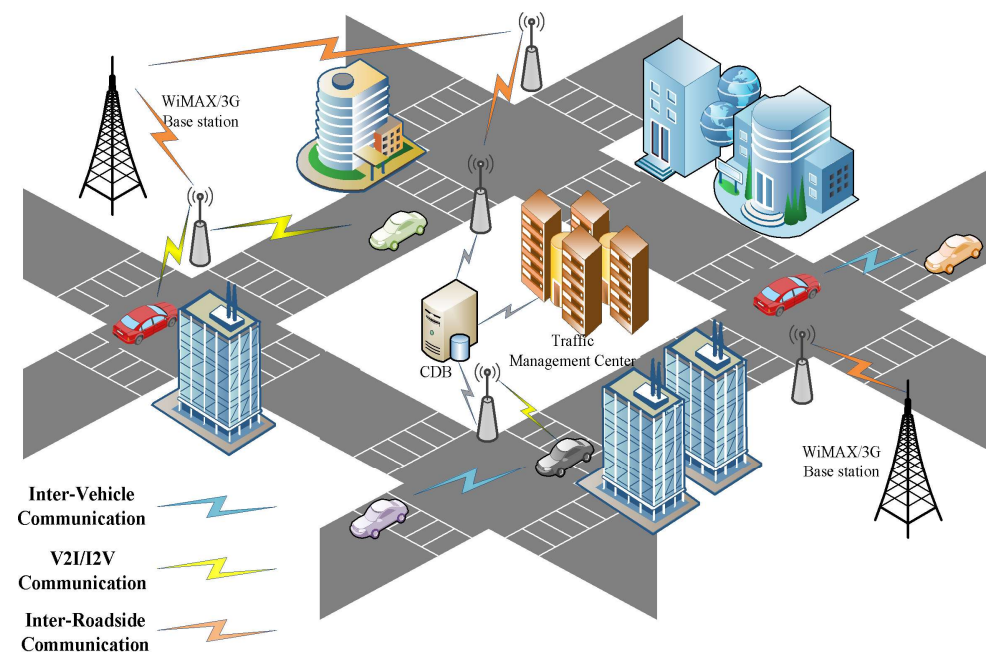

Fig. 2: Overview of VANET operation

The main contributions of this paper are as follows: 1. To provide an overview of the existing problems for $\mathrm{CPZ}$ systems. 2. To design an optimized CPZ model using VANET. 3. To compare and evaluate the performances of two communication methods (RFID and DSRC), whereby several traffic metrics such as the number, velocity, and occupancy of the contributed vehicles and traffic flow are considered. The rest of the paper is structured as follows: section 2 discusses the CP concept and the most important projects along with their shortcomings; section 3 elaborates on the detailed functionality and phases of the proposed system; section 4 includes the simulation results and system evaluation; and lastly, section 5 concludes the paper and suggests the direction for future research.

\subsection{LITERATURE REVIEW}

In this section, an overview of $\mathrm{CP}$ and its most relevant projects are discussed. Moreover, the problems of the existing projects are classified into the following two categories: system-design weaknesses and technological problems (with a focus on the data-communication phase).

\subsection{Congestion pricing $(\mathrm{CP})$}

Congestion pricing (CP), also known as "congestion charge," is one of the efficient-pricing strategies that have been offered by economists and transportation engineers to reduce the waste associated with traffic congestion [19]. The concept's principle requires users to pay for the use of some transportation restrictions. The CP contains the following different scheme types: facility-based, area-based, and distance/weight-based. Facilitybased CP is roughly a combination of CP techniques such as tolling systems, vehicle-access control, and eligibility restrictions [20]. Area-based CP designates a part of the urban environment as a congestion area and requires drivers to pay to access this restricted area [21]. For distance-based schemes, charges vary according to the distance traveled either linearly or nonlinearly. Additionally, some facilities charge on the basis of the weights that heavy-goods vehicles (HGVs) carry, and this type mostly applies in networks of truck-only toll lanes [22]. Since this paper is concentrated on an area-based CP scheme, the related explanations and categories are discussed in the following section.

Area-based CP projects, also known as CPZs, have been applied in several countries and their purposes besides congestion management, are emission reduction and governmental revenue collection. This type of $\mathrm{CP}$ contains 
two main subcategories; cordon schemes and zonal schemes that are subsequently described in this paper. In comparison with a facility-based scheme, the CPZ type has the advantage of intercepting more trips and is more suitable for traffic-congestion reduction [5].

Cordon Scheme - This scheme requires drivers to pay to cross a cordon in either the inbound or outbound direction, or possibly in both directions [23]. It is worth mentioning that all of the applied schemes are single cordons such as the Norwegian urban toll ring, Stockholm congestion charge, and Singapore ERP.

Zonal Scheme - This scheme forces drivers to pay to enter or exit a charging zone, or even to travel inside the zone without crossing its boundary. Charging-zone edges are definable by natural structures or by elements of the built environment. The London congestion charge is an example of this category [8].

As declared previously, the functioning of a CPZ system is based on the following three main phases: vehicle detection, data communication, and enforcement. The most well-known CPZ projects along with their functionalities are explained in the following section.

\subsection{Existing CPZ Systems}

London Congestion Charge [3], implemented in February 2003, charges any private vehicle for driving into a Congestion Charge Zone (CCZ) within the time period of 7:00 a.m. to 6:00 p.m. during weekdays. The CCZ comprises a circle with a $2.7 \mathrm{~km}$ radius in the London central-city area (around $22 \mathrm{~km}^{2}$ ). The main aim of this project is to reduce traffic congestion in the central-city area and to increase revenue collection for transportation improvements. Motorists are required to top up their accounts (vehicle's plate number is stored in a database) at selected retail outlets that are spread out in the vicinity of the congestion-charge areas; accordingly, since prepaid reloads are conducted through payment machines, vehicle profiles are updated in the traffic-management database. Generally, the enforcement procedure is based on the Automatic Number Plate Recognition (ANPR) system that is comprised of a vast network of video cameras that capture plate numbers and match them with the paid list [24]. Violation-enforcement notifications are sent to drivers' cell phones via cellular telephonemessaging technology. Economists believe that the London congestion charge has significantly reduced traffic congestion and emissions in the central-city area, has improved bus and taxi services, and generates substantial revenues.

Singapore's Electronic Road Pricing (ERP) [5] system, introduced in July 1998, is the first time that such a model has been implemented on such a wide scale to manage traffic congestion. The chief aim of the ERP is to alleviate congestion and increase traffic flow in a designated part of city (the restricted-zone amplitude is approximately 725 hectares) by imposing a charge on vehicles that are considered the main contributors to congestion. It is worth mentioning that the Singaporean government revises the charging schedule every three months, and vehicle charges are currently imposed from 7:00 a.m. to 9:30 a.m. and from 5:30 p.m. to 10:30 pm on weekdays only. The ERP system consists of the following three main components: gantries, in-vehicle units (IUs), and smart cards that are also known as "CashCard." Each gantry is equipped with two sets of antennas, two video cameras, and two optical detectors. Alternatively, an IU is a pocket-sized device that is permanently attached to a vehicle's windscreen to communicate with a gantry's antennas using an RFID. The third component refers to the smart cards that need to be charged by drivers and that become functional when they are embedded into an IU. Gantries are responsible for the detection of an incoming vehicle through communication with the IUs to identify the vehicle type and deduct the charging fee; also, in the case of a violation, they capture the vehicle's plate number. The gantries are connected to an outstation controller via a coaxial cable to report offences and charging procedures. All vehicle violations are mailed to the owners' addresses for consideration.

The Stockholm congestion tax [4] was publicly re-introduced on August 1, 2007. The charging zone features a cordon around the city center that is equipped with 18 control points that charge vehicles entering and exiting the charging zone from 6:30 a.m. to 6:30 p.m. on weekdays. The Swedish system uses a variable-fee pricing method that varies by vehicle type and time of day (increase during peak hours). Emergency vehicles, disabled people's vehicles, city buses, taxis, clean cars (vehicles that use alternative fuel), and foreign cars are exempt from being charged. The Stockholm congestion tax was previously used to take advantage of the RFID system to accomplish data communication. In 2007, however, it was decided that the system would be replaced by Automated Number Plate Recognition (ANPR) so that vehicle license-plate numbers could be captured. A monthly bill that is also available on the Internet is sent to the vehicle-owner's address. This system has managed to alleviate vehicle traffic ( $10 \%$ to $15 \%)$, has reduced traffic congestion inside the charging zone by 
$20 \%$, and decreased CO2 emissions (10\% to $14 \%$ ) [25]. Table 1 summarizes the specifications of the leading, functioning $\mathrm{CPZ}$ systems in terms of the exposure of the previously mentioned phases.

Table 1: Area-based CPZ scheme specifications

\begin{tabular}{|c|c|c|c|c|c|c|}
\hline \multirow{2}{*}{ Category } & \multirow{2}{*}{ Project } & \multirow{2}{*}{$\begin{array}{c}\text { Country } \\
\text { (Year) }\end{array}$} & \multicolumn{4}{|c|}{ Characteristics } \\
\hline & & & System type & Vehicle detection & Data Communication & Enforcement \\
\hline \multirow{3}{*}{$\begin{array}{l}\text { Cordon } \\
\text { Scheme }\end{array}$} & $\begin{array}{c}\text { Stockholm Congestion } \\
\text { Charge }[4]\end{array}$ & $\begin{array}{l}\text { Sweden } \\
(2007)\end{array}$ & Roadside-only & Video Cameras & RFID & ANPR \\
\hline & $\begin{array}{c}\text { Singapore ERP } \\
\text { (Electronic Road } \\
\text { Payment) } \\
{[5]}\end{array}$ & $\begin{array}{l}\text { Singapore } \\
(1998)\end{array}$ & $\begin{array}{l}\text { RSU \& IVU with } \\
\text { smartcards } \\
\text { (Tag and beacon) }\end{array}$ & RF beacon & RFID & ANPR \\
\hline & $\begin{array}{c}\text { Norwegian toll ring } \\
{[6]}\end{array}$ & $\begin{array}{l}\text { Norway } \\
(2004)\end{array}$ & $\begin{array}{l}\text { RSU \& AutoPass } \\
\text { (Tag and beacon) }\end{array}$ & Optical beacon & RFID & ANPR \\
\hline \multirow{2}{*}{$\begin{array}{l}\text { Zonal } \\
\text { Scheme }\end{array}$} & $\begin{array}{c}\text { London Congestion } \\
\text { Charge [3] }\end{array}$ & $\begin{array}{c}\text { United } \\
\text { Kingdom } \\
(2003) \\
\end{array}$ & Roadside-only & Video Cameras & - & ANPR \\
\hline & Proposed system & & $\begin{array}{l}\text { RSU \& OBU } \\
\text { (VANET) }\end{array}$ & $\begin{array}{c}\text { DSRC } \\
(\text { IEEE } 802.11 \mathrm{p})\end{array}$ & $\begin{array}{c}\text { DSRC } \\
(\text { IEEE } 802.11 \mathrm{p})\end{array}$ & $\begin{array}{c}\text { DSRC } \\
\text { (IEEE } 802.11 \mathrm{p})\end{array}$ \\
\hline
\end{tabular}

ANPR: Automatic Number-Plate Recognition, IVU: In Vehicle Units, RFID: Radio Frequency Identifier, RF-SRC: Radio-Frequency, Short-Range Communication

\subsection{Existing CPZ-system drawbacks}

Although the functionalities of existing systems have been developed over time, the systems still suffer from several deficiencies. In this section, CPZ problems are discussed in relation to the utilized technologies and system-design weaknesses; notably, the focus of this section is the communication-technology problems of CPZ systems.

\subsubsection{Technological problems}

Generally, the technologies of CPZ systems can be categorized as communicational and non-communicational technologies. Non-communicational technologies are mostly functioning in the first and second phases. The most popular non-communicational technology that is functioning in CPZ systems is ANPR. APNR is a vast network of video cameras for monitoring vehicles that is the most common mass-surveillance method in terms of violation enforcement for facility-based and area-based CP systems; however, ANPR is unable to perform data communication and needs a complimentary technology in this regard [26]. Besides advantages like an antitamper robustness, there are some problems regarding false or stolen license-plate numbers, and a low rate of detection accuracy in poor weather conditions [23]. In addition, since these cameras are mounted throughout city environments, people have identified them as an invasion of privacy. The performance of ANPR is lacking in terms of accuracy and reliability.

There are various methods that can accomplish the data-communication phase, including the optical beacon, spread spectrum radio, infrared beacon (IR), and radio frequency beacon (RF). These are different kinds of Short Range Communications (SRCs) that are applied in existing CP systems to perform the datacommunication phase [27]. RFID, which is a developed version of the RF spectrum, is the most utilized SRC type for various CPZ and tolling systems. Obviously, however, RFID also comprises several disadvantages as follows:

i. Transmission range and data rate

The most tangible disadvantages of RFID are its low signal-coverage distance and low data-rate propagation level, which are $10 \mathrm{~m}$ and $250 \mathrm{kbps}$, respectively. Since the RFID propagates packets in a series over a narrow channel, it causes low data-transmission speed. On the other hand, the transmission packets do not cover large amounts of data [28].

\section{ii. $\quad$ Signal fading and disruption}

Moreover, communication sessions are vulnerable to disruption and interception (even with encryption). In addition, signal fading, signal interference, and reading errors for antennas are common when using RFID due to 
the adverse conditions of the environment, the presence of metal obstacles, and the improper placement of tags, as well as absorption and an ambient reflection of the signal and external-signal sources (such as security systems, cordless phones, and barcode scanners) [29]. It is worth mentioning, that since most of the antennas that are used in tags are direction-sensitive, improper orientation of tags may impair reading efficiency.

\section{iii. Collisions}

Signal collision is another disadvantage of the RFID environment that results in data loss. Two types of collisions may occur while using RFID: tag collision and reader collision [30]. Tag collision happens in situations where a reader antenna has multiple vehicles within its coverage range (read range). In such situations, multiple tags are communicating with the RFID tag reader simultaneously; therefore, the reader is unable to differentiate these signals and data loss occurs. Reader collision occurs in situations where the coverage area of one RFID reader antenna overlaps with another. It can cause two different deficiencies. The first deficiency is signal interference from the overlapping and interfering of two RFID reader antennas. Secondly, each of the overlapping readers can enact multiple reads of the same tag. Particularly due to its shortrange read-range coverage, RFID is unable to operate under multiple overlapping communication zones where multiple tags exist in the reading zone (coverage area).

\section{iv. Tag limitations}

Tags are extremely vulnerable to damage, while the faulty manufacture of tags is $25 \%$ to $30 \%$ [28]. Another shortcoming of the RFID tag and beacon communication is that the tags cannot be controlled dynamically in terms of several factors such as emitted power, propagation channel, and message priority.

\section{v. Communication method}

RFID systems operate according to a master-slave communication method. Master-slave is a model of communication whereby one device has unidirectional control over other devices. In this case, the RFID reader antennas act as the controller (the master), and all of the tags on the vehicles act as the controlled devices (the slaves) [31]. In RFID systems, tags are read-only devices and a dynamic modification of a tag is not possible. In the current ITS field, peer-to-peer communication is compulsory because various applications operate in Infrastructure to Vehicle (I2V), Vehicle to Vehicle (V2V), and Vehicle to Infrastructure (V2I) communication types; that is, RSUs no longer act as solely a master device because vehicles can also act as the master in a number of cases. In some applications, the communication is only based on V2V communication and does not involve RSUs. Since RFID systems contain read-only tags as a part of communication devices, they cannot operate as a peer-to-peer communication technology [32]. A communication technology in the CPZ field is therefore missing, whereby the possibility of a peer-to-peer communication that allows drivers to interact with the CPZ system through a provided OBU interface in their vehicle is yet to be designed.

\subsubsection{System-design weaknesses}

CP schemes suffer from various deficiencies including payment method, loss of privacy, flexibility, and cost, which are described below:

\section{i. $\quad$ Payment method}

One of the main phases of a CP system is the charging and payment methods. There are many critics of the payment methods of current systems who argue that there are more cost-effective ways to collect money [2], where the advantages of vehicular networks and cloud computing are embraced.

\section{ii. Loss of privacy}

The majority of current CP systems perform the enforcement task using the ANPR mass-surveillance method. Accordingly, however, citizens' concerns emerged regarding the invasion of privacy that such networks of video cameras and systems for the tracking of vehicles within cities (especially London) represent [3].

\section{iii. Flexibility}

Most CP systems are not flexible enough to accommodate the variations in different issues such as the redeployment or expansion of the charging area (because the equipment cannot be relocated) and the 
modification of the system's functionality method, as instant application changes the charging fees [2]. Besides, adding new technology, salvaging old equipment, and adding services like route navigation are also not possible.

\section{iv. Cost}

The budgets of such projects not only refer to the operation of the equipment, but they also include the repair and maintenance costs regarding the whole system and infrastructures. The authors of [3] clarified that the expenses of $\mathrm{CP}$ systems that utilize video-camera roadside infrastructure is high; therefore, a new model needs to be proposed to decrease both the implementation and maintenance expenses.

To mitigate the discussed problems in the existing CPZ systems, an optimized CPZ model that takes advantage of VANET is proposed in section 3.

\subsection{PROPOSED MODEL}

This proposed system is an optimization model for managing a CPZ that performs the following three main tasks: 1. Initialization and verification 2. Charging 3. Violation enforcement. Each task is described in detail in the following subsections and are illustrated in Figure 3.

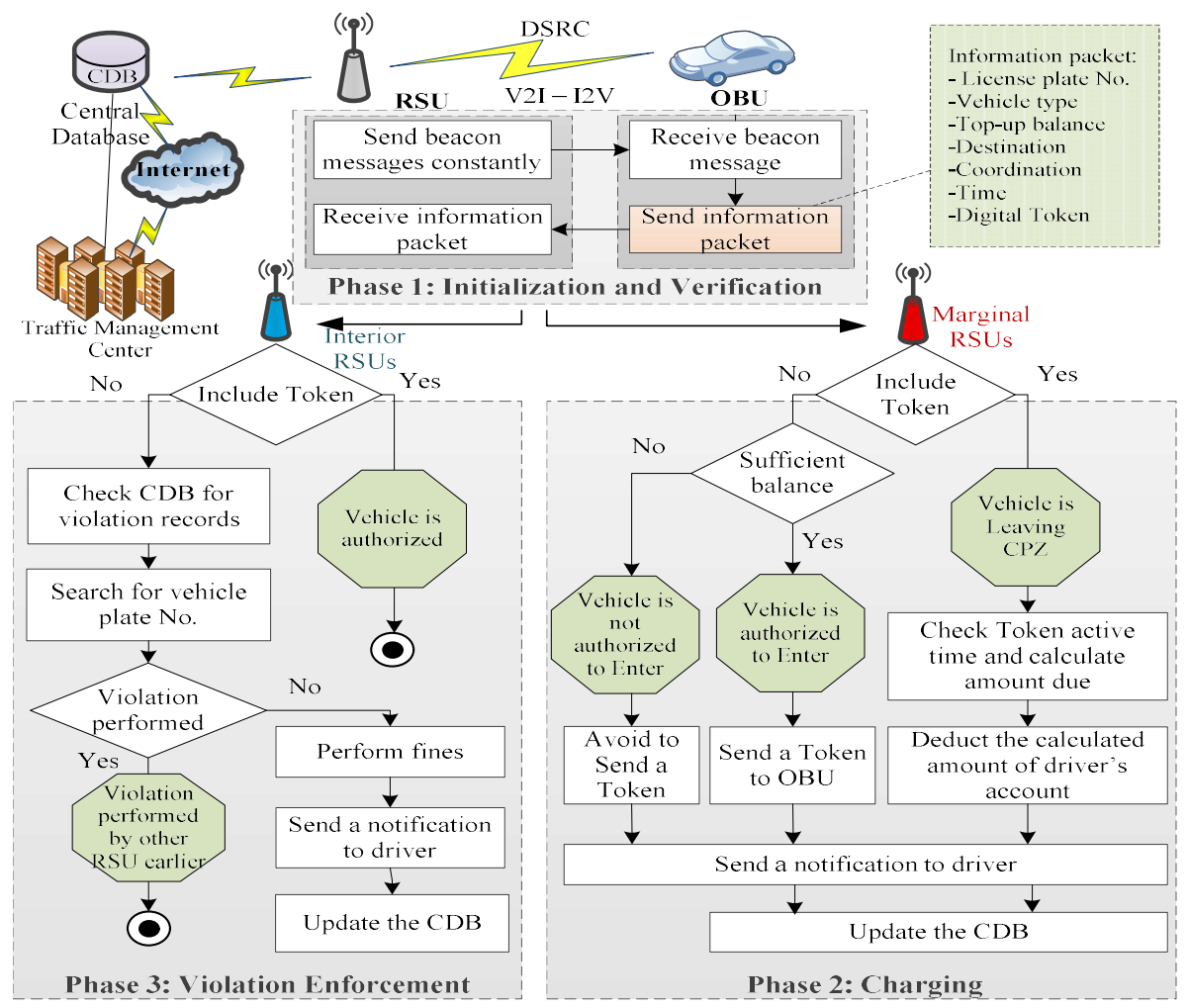

Fig. 3: General Optimization Model for Congestion Pricing Zone Management

Before explaining the proposed system's functionality, some prerequisites and assumptions need to be clarified before the system's processes are initiated. As the proposed model is a VANET-based system, all of the vehicles are equipped with OBU; in addition, a vast number of RSUs had been embedded all over the CPZ so that they covered all of the areas of CPZ. It is worth mentioning that this model comprises the following two types of RSU: 1. Marginal RSUs that are mounted at the edge of the CPZ and are responsible for charging and routing tasks. 2. Interior RSUs that are spread throughout the inside of the CPZ and are in charge of violation enforcement. Both types of RSUs are connected to a Central Data Base (CDB) that is directed toward the main traffic-management center. Accordingly, every vehicle has a CDB profile that contains their information 
including a vehicle license-plate number, vehicle type (passenger, truck, van, motorcycle), and top-up balance, destination, and/or token.

\section{1- Initialization and verification}

Initialization and verification are basilar levels of this system that refer to the communication process between a vehicle's OBU and RSUs. Particularly, RSUs need a vehicle's information for initiation and authentication. To do so, and for stablishing a session, both the marginal and interior RSUs constantly broadcast beacon messages in the range of $200 \mathrm{~m}$ (although DSRC antennas can cover up to $400 \mathrm{~m}$ ) and any vehicle under the coverage of their signal propagation can receive it. This session is a V2I/I2V communication type that is conducted via DSRC communication technology; therefore, OBU sends back a vehicle-information packet that comprises information about the vehicle license-plate number, vehicle type, top-up balance, destination, coordination, time, and/or digital token. The token is used to measure the charging fee and its functionality is described in the tasks of the following section. The RSU then receives the information packet and it is verified with CDB for validation. As mentioned earlier, vehicles' profiles are recorded in the CDB that is connected to the Traffic Management Centre. It is worth mentioning that if a foreigner's vehicle from another city intends to enter the $\mathrm{CPZ}$, since there is no available profile in the database for validation; RSU adds this vehicle to the CDB. Figure 3 illustrates the process of initialization and verification between the RSU and OBU.

\section{2-Charging}

First, it is worth noting that the marginal RSUs are responsible for performing the charging phase. After the accomplishment of the initialization and verification phases, the marginal RSUs look for a token inside the packet. Two possible conditions can occur, either the packet contains the token or it does not. In the case where the token does not exist, the vehicle intends to enter the $\mathrm{CPZ}$ and this is related to the charging phase. Alternatively, if the packet contains the token, the vehicle previously collected the token and now intends to exit the CPZ, and the case should be navigated via the routing phase. The active time of the token is calculated using the vehicle-packet information including entrance/exit time and coordination. Accordingly, the system calculates the amount due and deducts the fee from the vehicle's account; in addition, the system communicates with the $\mathrm{CDB}$ to record the transaction in the user's profile and also sends a notification referring the transaction to the driver. Fig. 4 shows a demonstration of the charging phase based on the following scenario: First, vehicle A (yellow car) reloads via OBU (through Internet) so that it is authorized to enter the CPZ (3-a); vehicle A moves closer to the CPZ so that it is possible for OBU to receive beacon messages from the marginal RSUs. After the initialization and verification phases are performed, vehicle A enters into the CPZ while marginal RSU checks for vehicle type, entrance time, coordination, and a sufficient balance from the corresponding CDB profile. Since this vehicle had a top-up before, the RSU sends a digital token to OBU (3-b). Whenever vehicle A intends to exit, the marginal RSU checks the token active time and calculates the due amount (3-c).
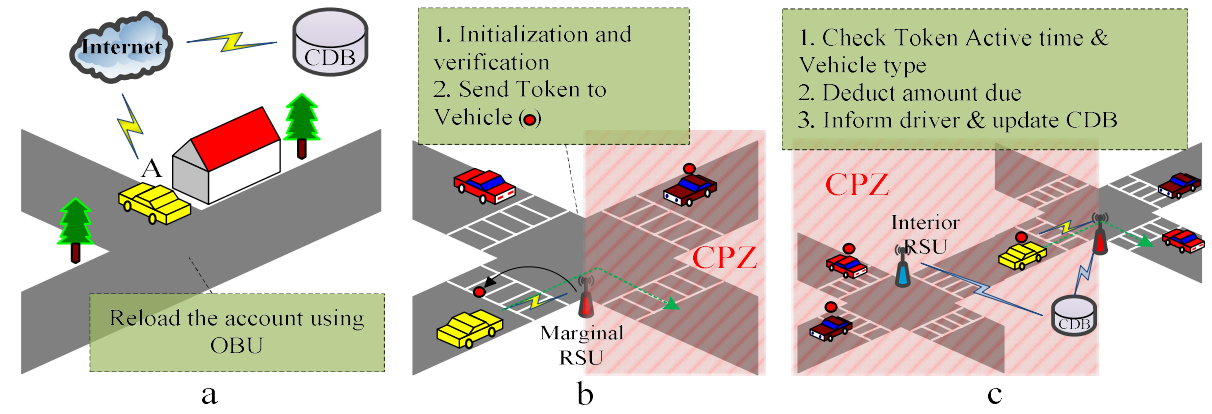

Fig. 4: Charging case scenario

\section{3-Violation}

Different to previous tasks, violation enforcement is under the interior RSUs' responsibility. After performing the initialization and verification phases, the interior RSUs search for the token inside the packet. As illustrated in Fig. 5, vehicle $\mathrm{C}$ will be located under the coverage of several interior RSUs while traveling within the CPZ. In the case where a token exists in the received packet, the process will be terminated, but when the RSUs find a vehicle without a token, they proceed to search license-plate numbers in the CDB violation-record list. Therefore, if the fine has been conducted before, the process will terminate the RSU's enforcement of the 
violation again; the CDB will be updated about this process to avoid further fines from other interior RSUs, and a notification will finally be sent to the driver.
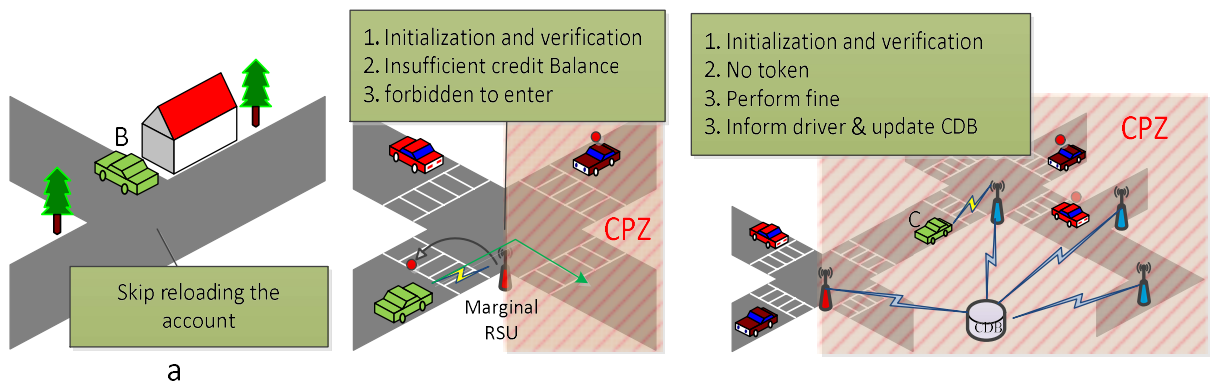

Fig. 5: Violation-enforcement case scenario

\subsection{PERFORMANCE EVALUATION}

In this section, the effects of using the two most well-known communication technologies-DSRC (IEEE 802.11p) and RFID - are evaluated in relation to a CPZ system and several traffic criteria such as the number, velocity, and occupancy of the contributed vehicles and the traffic flow. It is worth mentioning that, due to the popularity of the utilization-passive RFID in the ITS environment [10], the passive type of RFID is evaluated in this study; this evaluation was conducted in an intersection similar to the one depicted in Fig. 6. and in particular, one side of this junction is the CPZ entrance. The RSU is located in the corner of the intersection (CPZ-entrance side) that is used for the marginal RSU's communication with vehicles regarding the CPZ. The numbers of 50 vehicles were generated and they randomly entered into the CPZ through three shown paths and their traffic behavior was captured via an embedded loop detector at the CPZ entrance. This assessment has been conducted in a $600 \mathrm{ds}$ (decisecond) time span that is separated into the following four time intervals: 150 ds, $300 \mathrm{ds}, 450 \mathrm{ds}$, and $600 \mathrm{ds}$. As shown in Figure 6, the yellow spot is a loop detector that is responsible for measuring the previously mentioned traffic metrics. To accomplish our objective, this evaluation was conducted under two different conditions. Under the first condition, the marginal RSU is considered as an RFID transceiver, while in the second it is considered a DSRC transceiver.

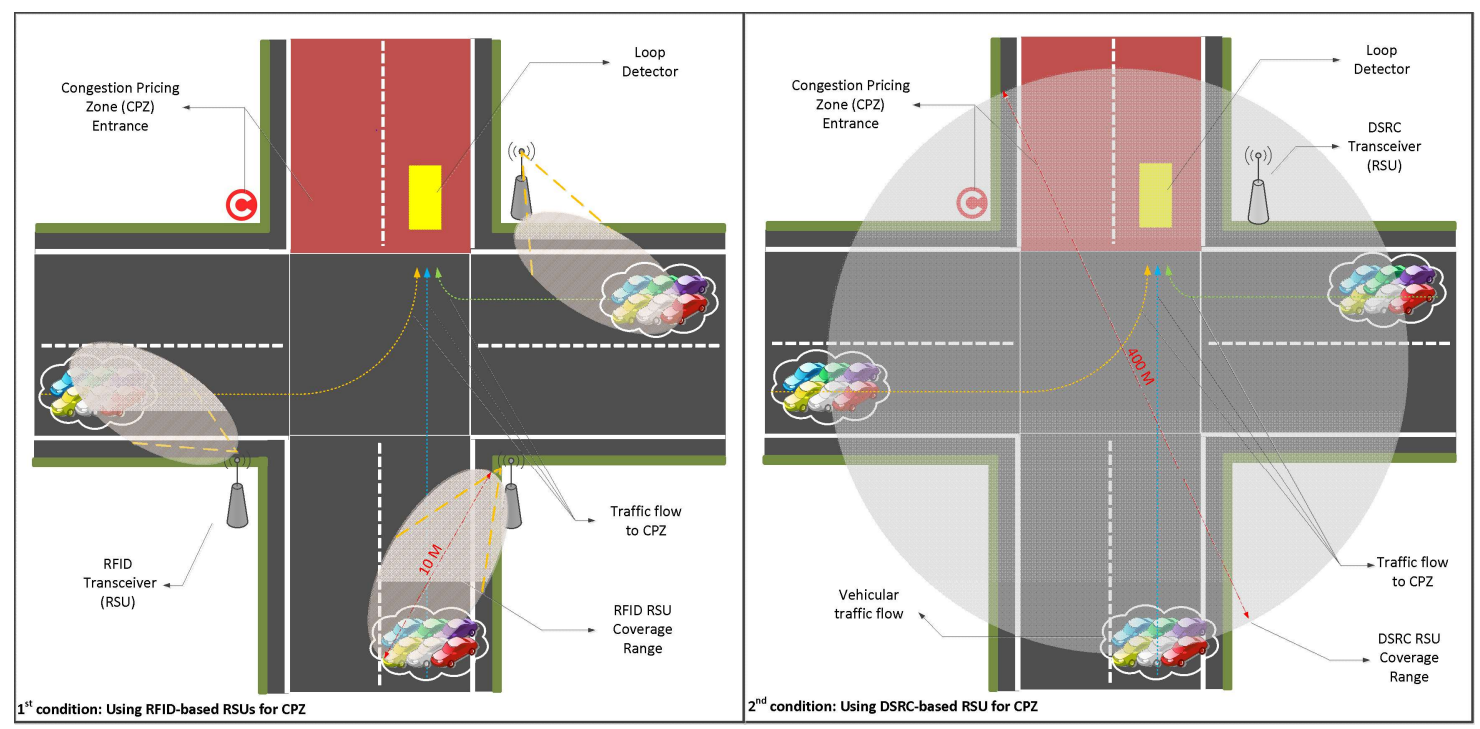

Fig. 6: Performance-evaluation scenario

The experiment was conducted in the Simulation of Urban Mobility (SUMO) [33], which is one of the most cited traffic-mobility simulators in recent, related VANET-based studies. SUMO provides a graphical user 
interface (GUI) in a $\mathrm{C}++$ programming environment to enable the creation of maps and traffic, as well as the provision of the ability to import real maps and a variety of geographical features to create more practical and efficient scenarios. The first 150 ds of the output from SUMO was discarded to get more accurate results. Table 2 summarizes the simulation parameters that were used for SUMO.

Table 2: Configuration parameters in SUMO simulation

\begin{tabular}{ll}
\hline Parameter & Value \\
\hline \hline Simulation Time & $600 \mathrm{ds}$ (decisecond) \\
Vehicle Density & 50 \\
Vehicle Initial Speed & $15 \mathrm{~m} / \mathrm{s}$ \\
Vehicle MaxSpeed & $20 \mathrm{~m} / \mathrm{s}$ \\
Vehicle Acceleration & $2.6 \mathrm{~m} / \wedge^{\mathrm{s}}$ \\
Vehicle Deceleration & $4.5 \mathrm{~m} / \wedge^{\mathrm{s}}$ \\
Vehicle Length & $5.0 \mathrm{~m}$ \\
minGap & $2.0 \mathrm{~m}$ \\
Speed Factor & 1.0 \\
Speed Deviation & 0.1 \\
Driver impatience & 0.6 \\
Sigma (driver imperfection) & 0.5 \\
\hline
\end{tabular}

Alternatively, a part of our proposed model was implemented in the NS-2.33 simulator [34]. At the MAC and physical layers, the IEEE 802.11p standard (DSRC) [16] and RFID were configured as wireless communications. The maximum transmission range for the RFID and DSRC were set to $10 \mathrm{~m}$ and $400 \mathrm{~m}$, respectively. Table 3 summarizes the simulation parameters that were used for NS-2. Traffic Control Interface (TraCI) [35] was used to interlink the vehicle mobility generator (i.e. SUMO) and the network simulator (i.e. NS-2) to control the vehicle's mobility and traffic behavior during the simulation runtime. The TraCI provides this interaction over a TCP connection. The TraCI utilizes a client/server based architecture in which the SUMO and NS-2 software play the role of TraCI-server and TraCI-client, respectively. After establishing a TCP connection, the TraCI-client (NS-2) uses atomic mobility commands (i.e. commands and responses) to control the simulated vehicle movements in the TraCI server (SUMO) that is based on our proposed model under the two previously mentioned conditions. It is worth mentioning that TraCI is one of the features of the latest version of TraNS (TraNS v1.2) [36]. All of the results represent an average of over 25 executions with different scenarios.

Table 3: Configuration parameters in NS-2 simulation

\begin{tabular}{ll}
\hline Parameter & Value \\
\hline \hline Simulation Time & 600 ds (decisecond) \\
Mac/PHY & a. DSRC (IEEE802.11p) \\
& b. RFID (Active mode) \\
Max. Transmission Range & a. DSRC $-400 \mathrm{~m}$ \\
& b. RFID $-10 \mathrm{~m}$ \\
Max packet size & $250 \mathrm{~kb}$ \\
Mobility Generator & SUMO \\
\hline
\end{tabular}

\subsection{ASSESSMENT}

The communication between RSU and OBU required the establishment of an I2V session for conducting the authentication and authorization, as well as for sending and receiving information packets between RSU and OBU. Obviously, like any other procedure this process is time consuming, with the time durations vary depending on the kind of communication technology that is being used. Although this communication needs time span for conduction, this amount can also change a vehicle's behaviour, which has a direct impact on roadtraffic results; that is, vehicles need to decrease their velocity to make communication possible between RSU and OBU. 
For this section, and according to the scenario map, the obtained results from the loop detector regarding the vehicles' traffic-behaviour information from the CPZ entrance, under two different conditions (RFID-based RSU and DSRC-based RSU), were taken for analysis and various evaluation metrics were considered. The number, velocity, and occupancy of the contributed vehicles and the traffic flow are four evaluation metrics that are used to evaluate the effects of SRC and RFID in a CPZ system. The previously mentioned traffic-evaluation criteria are analysed in the following section to conclude which communication technology is more suitable in the field of CPZ.

Traffic density is one of the most essential factors that needs to be considered to evaluate the traffic congestion in an area. In our particular scenario, the number of contributed vehicles through the CPZ entrance was evaluated. The information about vehicle density was gathered from an embedded loop detector at the $\mathrm{CPZ}$ entrance. The number of contributed vehicles at the $\mathrm{CPZ}$ was evaluated at four separated time intervals containing $150 \mathrm{ds}, 300 \mathrm{ds}, 450 \mathrm{ds}$, and $600 \mathrm{ds}$. Fig. 7 illustrates that, by increasing the simulation time, the number of contributed vehicles decreased smoothly. Although the number of contributed vehicles was reduced using both the RFID and DSRC technologies, DSRC had a higher rate of passed vehicles at every single time interval.

Traffic flow is the main metric to evaluate traffic congestion, which expresses the number of contributing vehicles extrapolated in a time period of $60 \mathrm{~min}$ (number of vehicles per hour). Figure 7 illustrates the float ratio of the hourly number of passed vehicles through the loop detector at the CPZ entrance within the four separate time intervals of $150 \mathrm{ds}, 300 \mathrm{ds}, 450 \mathrm{ds}$ and $600 \mathrm{ds}$. As the diagram shows, by increasing the time span of the simulation, the traffic flow was reduced gradually under both RFID and DSRC.

The decrement of traffic flow over the passing of time is a mean of congestion but this assessment aims to show which technology contributes to less congestion in the CPZ vicinity. As the results depict, DSRC caused a higher rate of traffic flow in comparison with RFID. After passing $300 \mathrm{ds}$, the minimum difference between using DSRC and RFID is 200 (NoV/Hr), which is impressive; consequently, we concluded that the utilization of DSRC improved the traffic flow by an average of $22 \%$, which is the mean of less congestion and the provision of better traffic flow at the edge of the CPZ.

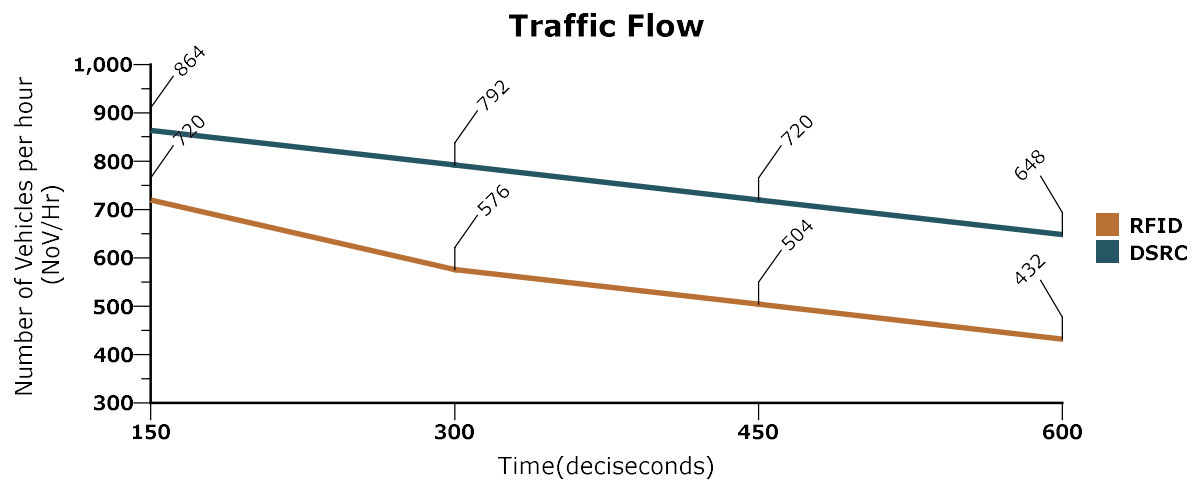

Fig. 7: Traffic flow using RFID and DSRC

Another important metric to assess traffic congestion in a designated area is occupancy. This metric indicates the percentage of the time that a vehicle occupies the loop detector; therefore, higher occupancy means that the time duration that a vehicle occupied the loop detector is higher, resulting in congestion. A lower occupancy means less congestion, creating the ideal condition; accordingly, the vehicle's occupancy depicted in Fig. 8 assesses two different conditions using RFID and DSRC, and the result shows that occupancy has a direct relationship with the passing of time. A comparison between the first time period ( $150 \mathrm{ds}$ to $300 \mathrm{ds})$ and the last time period (450 ds to $600 \mathrm{ds}$ ) verifies that occupancy under the RFID condition was dramatically increased. Nonetheless, the occupancy rate under the DSRC condition also increased, but it is $8.27 \%$ lower than the RFID. 


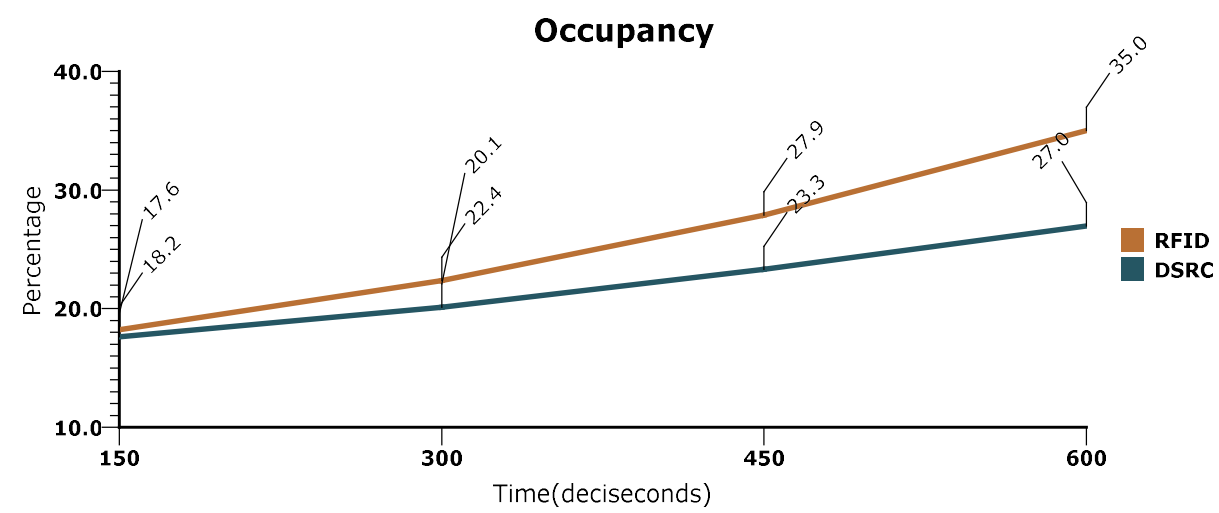

Fig. 8: Occupancy using RFID and DSRC

A vehicle's velocity counts is one of the key criterions for traffic assessment. The velocity is the instant speed of all of the vehicles that completely pass the loop detector. The higher a vehicle's average velocity rate, the lower the rate of traffic congestion. Fig. 9 represents the average travel velocity of vehicles over the four separate time intervals of $150 \mathrm{ds}, 300 \mathrm{ds}, 450 \mathrm{ds}$, and $600 \mathrm{ds}$. As mentioned in Table 2, a vehicle's initial velocity at the beginning of the simulation time was assigned as $15 \mathrm{~m} / \mathrm{s}$. The results show that a vehicle's average speed has an inverse relationship with the passing of time, whereby the average vehicle speed decreases as the simulation time increases. According to the initial assigned speed $(15 \mathrm{~m} / \mathrm{s})$, the first time intervals were recorded as 13.6 $\mathrm{m} / \mathrm{s}$ and $10.96 \mathrm{~m} / \mathrm{s}$ for DSRC and RFID, respectively; therefore, in the first time interval, the vehicle's average speed decreased $13.34 \%$ and $33.34 \%$ under the DSRC and RFID conditions, respectively. In addition, the last time interval $(600 \mathrm{ds})$ shows that the vehicle's average speed under RFID decreased by $66.67 \%$, while it decreased by only $36.67 \%$ with DSRC. As a consequence, DSRC provided better performance in all of the time intervals with a higher average vehicle speed and a percentage of $13.75 \%$.

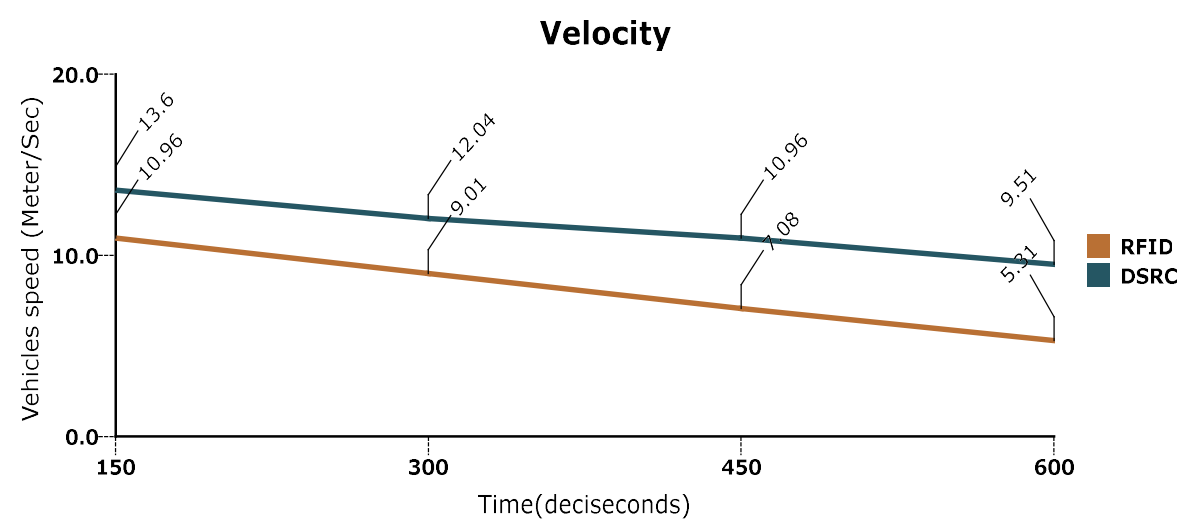

Fig.9: Vehicles average velocity using RFID and DSRC

\subsection{Conclusion and future work}

This study concentrated on area-based CP and includes a brief review of the CP concept, related implemented systems, different technologies, functionalities, and the pros and cons. Based on identified problems and limitations, we proposed a general VANET-based, CPZ-management model to take advantage of DSRC in VANET, we sought to address the technology weaknesses and system deficiencies of the existing systems. This proposed model seeks to optimize the traffic flow at the edge of a CPZ and to enhance data-communication performance, and it performs the three main tasks of initialization and verification, charging, and violation 
enforcement through DSRC-based RSUs. The SUMO, TraNS, and NS-2 simulator tools were utilized to implement our proposed model in a simulation environment. The traffic effects from using RFID as the most popular communication method in CPZ systems and DSRC (IEEE 802.11p) as a VANET communication type are evaluated in the same CPZ scenario map and concern several traffic criteria such as the number, velocity, and occupancy of the contributed vehicles and the traffic flow. The results show that DSRC outperformed the RFID in all of the previously mentioned traffic metrics, as it provided a higher bandwidth capacity, a higher transmission range, a higher data rate, and a lower delay. Based on the results, the number of contributed vehicles and traffic flow increased by an average of $22 \%$, occupancy decreased by an average of $8.27 \%$, and the velocity rate increased by an average of $13.75 \%$. In conclusion, the employment of DSRC under the VANET infrastructure not only enhanced data communication but it also caused a dramatic improvement in the traffic congestion around a CPZ. For future works, we are aiming to expand the simulation experiment by using some quality-of-service metrics such as communication latency, throughput, and packet loss in terms of different conditions like vehicle speed, density, communication bandwidth, and packet size. In addition, it is necessary to work on the corresponding communications' security aspects since the packets contain the private information of drivers.

\section{Acknowledgment}

This research is supported by a UM High-Impact Research MoE Grant UM.C/625/1/HIR/MOHE/FCSIT/09 from the Ministry of Education Malaysia and PRG-1436-16.

\section{REFERENCES}

[1] G. Duranton and M. A. Turner, "Urban growth and transportation," The Review of Economic Studies, vol. 79, pp. 1407-1440, 2012.

[2] M. H. TALUKDAR, "Framework for Traffic Congestion Management," Economia. Seria Management, vol. 16, pp. 54-64, 2013.

[3] G. Santos, K. Button, and R. G. Noll, "London congestion charging," Brookings-Wharton Papers on Urban Affairs, pp. 177-234, 2008.

[4] M. Börjesson, J. Eliasson, M. B. Hugosson, and K. Brundell-Freij, "The Stockholm congestion charges -5 years on. Effects, acceptability and lessons learnt," Transport Policy, vol. 20, pp. 1-12, 2012 .

[5] M. M. Haque, H. C. Chin, and A. K. Debnath, "Sustainable, safe, smart-three key elements of Singapore's evolving transport policies," Transport Policy, vol. 27, pp. 20-31, 2013.

[6] J. Odeck and A. Kjerkreit, "Evidence on users' attitudes towards road user charges - A cross-sectional survey of six Norwegian toll schemes," Transport Policy, vol. 17, pp. 349-358, 2010.

[7] Y. Yin and Y. Lou, "Dynamic tolling strategies for managed lanes," Journal of Transportation Engineering, vol. 135, pp. 45-52, 2009.

[8] A. de Palma and R. Lindsey, "Traffic congestion pricing methodologies and technologies," Transportation Research Part C: Emerging Technologies, vol. 19, pp. 1377-1399, 2011.

[9] R. Want, "An introduction to RFID technology," Pervasive Computing, IEEE, vol. 5, pp. 25-33, 2006.

[10] E.-K. Lee, S. Y. Oh, and M. Gerla, "RFID assisted vehicle positioning in VANETs," Pervasive and Mobile Computing, vol. 8, pp. 167-179, 2012.

[11] S. Al-Sultan, M. M. Al-Doori, A. H. Al-Bayatti, and H. Zedan, "A comprehensive survey on vehicular Ad Hoc network," Journal of network and computer applications, vol. 37, pp. 380-392, 2014. 
[12] Rasheed, A., Amjal, S., and Qayyum, A, "Adaptive Routing Update Approach for VANET Using Local Neighbourhood Change Information", Malaysian Journal of Computer Science, Vol. 27, Issue 4, pp. 307-327, 2014.

[13] A. Marefat, R. Aboki, A. Jalooli, E. Shaghaghi, M. R. Jabbarpour, and R. Md Noor, "An adaptive overtaking maneuver assistant system using VANET," in Wireless and Mobile, 2014 IEEE Asia Pacific Conference on, 2014, pp. 316-321.

[14] M. R. J. Sattari, R. M. Noor, and S. Ghahremani, "Dynamic congestion control algorithm for vehicular ad hoc networks," International Journal of Software Engineering and Its Applications, vol. 7, pp. 95$108,2013$.

[15] Y. J. Li, "An overview of the DSRC/WAVE technology," in Quality, Reliability, Security and Robustness in Heterogeneous Networks, ed: Springer, 2012, pp. 544-558.

[16] D. Jiang and L. Delgrossi, "IEEE 802.11 p: Towards an international standard for wireless access in vehicular environments," in Vehicular Technology Conference, 2008. VTC Spring 2008. IEEE, 2008, pp. 2036-2040.

[17] M. R. Jabbarpour, R. M. Noor, R. H. Khokhar, and C.-H. Ke, "Cross-layer congestion control model for urban vehicular environments," Journal of Network and Computer Applications, vol. 44, pp. 1-16, 2014.

[18] A. Jalooli, E. Shaghaghi, M. R. Jabbarpour, R. Md Noor, H. Yeo, and J. J. Jung, "Intelligent advisory speed limit dedication in highway using VANET," The Scientific World Journal, vol. 2014, 2014.

[19] R. Lindsey, "Road pricing and investment," Economics of transportation, vol. 1, pp. 49-63, 2012.

[20] T. Tsekeris and S. Vo $\beta$, "Design and evaluation of road pricing: state-of-the-art and methodological advances," Netnomics, vol. 10, pp. 5-52, 2009.

[21] S.-G. Yu, C.-M. Jeong, and H.-J. Lee, "Comparison of Area Pricing and Cordon Pricing in General Equilibrium Models," Journal of Korean Society of Transportation, vol. 27, pp. 145-155, 2009.

[22] Y. Lou, Y. Yin, and J. A. Laval, "Optimal dynamic pricing strategies for high-occupancy/toll lanes," Transportation Research Part C: Emerging Technologies, vol. 19, pp. 64-74, 2011.

[23] Z. Liu, Q. Meng, and S. Wang, "Speed-based toll design for cordon-based congestion pricing scheme," Transportation Research Part C: Emerging Technologies, vol. 31, pp. 83-98, 2013.

[24] G. Lekhana and R. Srikantaswamy, "Real time license plate recognition system," International Journal of Advanced Technology \& Engineering Research, vol. 2, pp. 5-9, 2012.

[25] R. Arnold, V. C. Smith, J. Q. Doan, R. N. Barry, J. L. Blakesley, P. T. DeCorla-Souza, et al., "Reducing congestion and funding transportation using road pricing in Europe and Singapore," 2010.

[26] A. Roy and D. P. Ghoshal, "Number Plate Recognition for use in different countries using an improved segmentation," in Emerging Trends and Applications in Computer Science (NCETACS), 2011 2nd National Conference on, 2011, pp. 1-5.

[27] M. L. Sichitiu and M. Kihl, "Inter-vehicle communication systems: a survey," Communications Surveys \& Tutorials, IEEE, vol. 10, pp. 88-105, 2008.

[28] M. Kaur, M. Sandhu, N. Mohan, and P. S. Sandhu, "RFID technology principles, advantages, limitations \& its applications," International Journal of Computer and Electrical Engineering, vol. 3, pp. 151-157, 2011. 
[29] F. Nekoogar and F. Dowla, "Characteristics and Limitations of Conventional RFIDs," in UltraWideband Radio Frequency Identification Systems, ed: Springer, 2012, pp. 25-49.

[30] X. Zhu, S. K. Mukhopadhyay, and H. Kurata, "A review of RFID technology and its managerial applications in different industries," Journal of Engineering and Technology Management, vol. 29, pp. 152-167, 2012.

[31] P. V. Nikitin, S. Ramamurthy, R. Martinez, and K. Rao, "Passive tag-to-tag communication," in RFID (RFID), 2012 IEEE International Conference on, 2012, pp. 177-184.

[32] X. Jia, Q. Feng, T. Fan, and Q. Lei, "RFID technology and its applications in Internet of Things (IoT)," in Consumer Electronics, Communications and Networks (CECNet), 2012 2nd International Conference on, 2012, pp. 1282-1285.

[33] M. Behrisch, L. Bieker, J. Erdmann, and D. Krajzewicz, "SUMO-Simulation of Urban MObility," in The Third International Conference on Advances in System Simulation (SIMUL 2011), Barcelona, Spain, 2011.

[34] T. Issariyakul and E. Hossain, Introduction to network simulator NS2: Springer Science \& Business Media, 2011.

[35] A. Wegener, M. Piórkowski, M. Raya, H. Hellbrück, S. Fischer, and J.-P. Hubaux, "TraCI: an interface for coupling road traffic and network simulators," in Proceedings of the 11th communications and networking simulation symposium, 2008, pp. 155-163.

[36] M. Piorkowski, M. Raya, A. L. Lugo, P. Papadimitratos, M. Grossglauser, and J.-P. Hubaux, "TraNS: realistic joint traffic and network simulator for VANETs," ACM SIGMOBILE Mobile Computing and Communications Review, vol. 12, pp. 31-33, 2008. 